\title{
Week 48 Resistance Analyses of the Once-Daily, Single-Tablet Regimen Darunavir/Cobicistat/Emtricitabine/Tenofovir Alafenamide (D/C/F/TAF) in Adults Living with HIV-1 from the Phase III Randomized AMBER and EMERALD Trials
}

\author{
Erkki Lathouwers, ${ }^{1}$ Eric Y Wong, ${ }^{2}$ Kimberley Brown,, Bryan Baugh, ${ }^{3}$ Anne Ghys, \\ John Jezorwski, ${ }^{4}$ El Ghazi Mohsine, Erika Van Landuyt, ${ }^{1}$ Magda Opsomer, ${ }^{1}$ and Sandra De Meyer, \\ on behalf of the AMBER and EMERALD Study Groups
}

\begin{abstract}
Darunavir/cobicistat/emtricitabine/tenofovir alafenamide (D/C/F/TAF) 800/150/200/10 $\mathrm{mg}$ is being investigated in two Phase III trials, AMBER (NCT02431247; treatment-naive adults) and EMERALD (NCT02269917; treatmentexperienced, virologically suppressed adults). Week 48 AMBER and EMERALD resistance analyses are presented. Postbaseline samples for genotyping/phenotyping were analyzed from protocol-defined virologic failures (PDVFs) with viral load (VL) $\geq 400$ copies/mL at failure/later time points. Post hoc analyses were deep sequencing in AMBER, and HIV-1 proviral DNA from baseline samples (VL $<50$ copies $/ \mathrm{mL}$ ) in EMERALD. Through week 48 across both studies, no darunavir, primary PI, or tenofovir resistance-associated mutations (RAMs) were observed in HIV-1 viruses of 1,125 participants receiving D/C/F/TAF or 629 receiving boosted darunavir plus emtricitabine/ tenofovir-disoproxil-fumarate. In AMBER, the nucleos(t)ide analog reverse transcriptase inhibitor (N(t)RTI) RAM M184I/V was identified in HIV-1 of one participant during D/C/F/TAF treatment. M184V was detected pretreatment as a minority variant (9\%). In EMERALD, in participants with prior VF and genoarchive data $(N=140 ; 98$ $\mathrm{D} / \mathrm{C} / \mathrm{F} / \mathrm{TAF}$ and 42 control), $4 \%$ had viruses with darunavir RAMs, 38\% with emtricitabine RAMs, mainly at position 184 (41\% not fully susceptible to emtricitabine), $4 \%$ with tenofovir RAMs, and $21 \% \geq 3$ thymidine analogassociated mutations (24\% not fully susceptible to tenofovir) detected at screening. All achieved VL $<50$ copies $/ \mathrm{mL}$ at week 48 or prior discontinuation. D/C/F/TAF has a high genetic barrier to resistance; no darunavir, primary PI, or tenofovir RAMs were observed through 48 weeks in AMBER and EMERALD. Only one postbaseline M184I/V RAM was observed in HIV-1 of an AMBER participant. In EMERALD, baseline archived RAMs to darunavir, emtricitabine, and tenofovir in participants with prior VF did not preclude virologic response.
\end{abstract}

Keywords: darunavir/cobicistat/emtricitabine/TAF, single-tablet regimen, resistance, efficacy, deep sequencing, archived RAMs

Introduction

W ITH THE INCREASING CHOICE of combination HIV-1 antiretroviral therapy (ART) regimens with similar high efficacy, selection of an ART regimen is frequently based on safety, tolerability, convenience, and genetic barrier to ART resistance. ${ }^{1-3}$ Understanding the resistance profile of the component antiretrovirals and the potential for emergence of resistanceassociated mutations (RAMs) is important, as this is likely to influence the extent of treatment and future treatment options. ${ }^{1-3}$

\footnotetext{
${ }^{1}$ Janssen Pharmaceutica NV, Beerse, Belgium.

${ }^{2}$ Janssen Scientific Affairs, LLC, Titusville, New Jersey.

${ }^{3}$ Janssen Research \& Development LLC, Raritan, New Jersey.

${ }^{4}$ Janssen Research \& Development, Pennington, New Jersey.

Data were presented previously in part at the National AIDS Treatment Advocacy Project, HIV Glasgow, 28 -31 October, 2018.
}

(C) Erkki Lathouwers et al. 2019; Published by Mary Ann Liebert, Inc. This Open Access article is distributed under the terms of the Creative Commons License (http://creativecommons.org/licenses/by/4.0), which permits unrestricted use, distribution, and reproduction in any medium, provided the original work is properly cited. 
Since its approval in 2006, a wealth of clinical trial data and clinical experience has been generated for boosted darunavir (DRV), demonstrating its durable virologic response, high genetic barrier to resistance, and long-term safety in a broad range of patients from ART-naive to highly treatment experienced. $^{4-11}$ The once-daily, single-tablet regimen (STR) of darunavir/cobicistat/emtricitabine/tenofovir alafenamide (D/C/F/TAF) 800/150/200/10 $\mathrm{mg}$ is approved in the United States, Europe, and Canada ${ }^{12,13}$ and is being investigated in two international, randomized Phase III trials, AMBER in ART-naive adults ${ }^{10}$ and EMERALD in ARTexperienced, virologically suppressed adults living with HIV$1^{11} \mathrm{D} / \mathrm{C} / \mathrm{F} / \mathrm{TAF}$ had noninferior efficacy versus $\mathrm{D} / \mathrm{C}$ plus emtricitabine/tenofovir disoproxil fumarate (F/TDF) (week 48 virologic response, viral load [VL] $<50$ copies/mL: $91.4 \%$ vs. $88.4 \%$, respectively; FDA-snapshot analysis) in $\mathrm{AMBER}^{10}$ and versus boosted protease inhibitor (bPI) + F/TDF for the primary efficacy outcome of protocol-defined virologic rebound (PDVR) cumulative through week 48 in EMERALD (D/C/F/TAF: $2.5 \%$ vs. bPI + F/TDF: $2.1 \%) .{ }^{11}$ Week 48 virologic failure (VF, FDA snapshot; VL $\geq 50$ copies $/ \mathrm{mL}$ ) was $0.8 \%$ versus $0.5 \%$, respectively, and virologic response was $94.9 \%$ versus $93.7 \% .^{11}$ Current treatment guidelines include DRV and cobicistat combined with two nucleoside or nucleotide analog reverse transcriptase inhibitors $(\mathrm{N}(\mathrm{t}) \mathrm{RTIs})$, or administered as the D/C/F/TAF STR, as a recommended treatment option, ${ }^{2}$ or recommended in certain clinical situations, such as for those patients who may have uncertain adherence, those who require a regimen with a high genetic barrier to resistance, or those patients who may not have resistance results available. ${ }^{1,3}$

We report HIV-1 resistance data in participants with protocol-defined virologic failure (PDVF) in AMBER and in EMERALD (participants with PDVR). In addition, HIV-1 proviral DNA from baseline samples (VL $<50$ copies $/ \mathrm{mL}$ ) was retrospectively analyzed to assess the prevalence of archived RAMs in participants with prior VF or PDVR, who were most likely to have baseline resistance, in EMERALD.

\section{Methods}

\section{AMBER and EMERALD study designs and participants}

Detailed methods for the international, multicenter, Phase III, randomized, active-controlled noninferiority trials, AMBER (TMC114FD2HTX3001; NCT02431247) ${ }^{10}$ and EMERALD (TMC114IFD3013; NCT02269917), ${ }^{11}$ have been reported previously.

AMBER (double-blind) included ART-naive adults with a screening plasma VL $\geq 1,000$ copies $/ \mathrm{mL}, \mathrm{CD}^{+}$cell count $>50$ cells $/ \mathrm{mm}^{3}$, and HIV-1 virus with genotypic sensitivity to DRV, emtricitabine, and tenofovir. EMERALD (open-label) included ART-experienced, virologically suppressed adults (VL $<50$ copies/mL for $\geq 2$ months before screening; one $50 \leq$ VL $<200$ copies/mL within 12 months before screening allowed) while on a stable bPI regimen (DRV/ritonavir or DRV/ cobicistat once daily, atazanavir/ritonavir or atazanavir/ cobicistat once daily, or lopinavir/ritonavir twice daily) + $\mathrm{F} / \mathrm{TDF}$ regimens for $\geq 6$ months. Patients with prior experience with multiple antiretrovirals and prior VF were eligible for study participation, provided they had no previous VF on DRV-based regimens and absence of DRV RAMs, ${ }^{14}$ if historical genotypes were available. No restriction was placed on the presence of emtricitabine or tenofovir RAMs or any other RAMs.
The primary outcome of both studies was to demonstrate noninferior efficacy of D/C/F/TAF 800/150/200/10 mg once daily compared with the control arm (bPI + F/TDF 200/ $300 \mathrm{mg}$ fixed-dose combination [FDC] in EMERALD or D/C 800/150 mg FDC + F/TDF 200/300 mg FDC in AMBER) at week 48. Patients continued receiving $\mathrm{D} / \mathrm{C} / \mathrm{F} / \mathrm{TAF}$ beyond week 48, provided they consented and continued to derive benefit as assessed by the investigator. Primary outcome measures were the proportion of participants with $\mathrm{VL}<50$ copies/mL (virologic response rate) by the FDA-snapshot analysis at week 48 (AMBER) or PDVR (confirmed VL $\geq 50$ copies/mL or premature discontinuations, irrespective of reason with last $\mathrm{VL} \geq 50$ copies/mL) cumulative through week 48 (EMERALD). The FDA-snapshot virologic response and VF rate (VL $\geq 50$ copies/mL) were also assessed as efficacy endpoints in EMERALD.

\section{Virology assessments}

In both studies, plasma HIV-1 RNA was quantified at screening, baseline, weeks $2,4,8$, and 12 , and every 12 weeks thereafter with the COBAS ${ }^{\circledR}$ AmpliPrep/COBAS ${ }^{\circledR}$ TaqMan HIV-1 Test V2.0 (Roche Diagnostics, Basel, Switzerland). Standard resistance testing was done when HIV-1 RNA was $\geq 400$ copies $/ \mathrm{mL}$.

ART-naive participants (AMBER). Genotypic resistance testing was performed using GenoSure ${ }^{\circledR}$ MG (HIV-1 protease [PR]/reverse transcriptase [RT] genotype assay; Monogram Biosciences, South San Francisco, CA) at screening. Genotypic and phenotypic testing was performed using PhenoSense ${ }^{\circledR}$ GT (combined HIV-1 PR/RT genotype/phenotype) postbaseline in participants with PDVF with VL $\geq 400$ copies/mL at failure (preferably confirmed, or at unconfirmed) or at later time points. ${ }^{10}$ PDVF was defined as virologic nonresponse $\left(\mathrm{VL}<1 \log _{10}\right.$ reduction from baseline and $\geq 50$ copies/mL at week 8 , confirmed at next visit) or virologic rebound (confirmed VL $\geq 50$ copies/mL after confirmed, consecutive $\mathrm{VL}<50$ copies/mL or confirmed $\mathrm{VL}>1 \log _{10}$ increase from the nadir) and/or viremia at the final time point (VL $\geq 400$ copies/mL at study endpoint or study discontinuation after week 8).

Deep sequencing using NGS GenoSure MG (Illumina MiSeq; codon variants $>1 \%$ ) was performed retrospectively on samples from participants with HIV-1 viruses that showed emerging resistance-associated mutations.

Virologically suppressed ART-experienced participants (EMERALD). Postbaseline HIV-1 PR and RT genotypic resistance testing was performed using GenoSure MG for participants with PDVR (confirmed VL $\geq 50$ copies/mL or single unconfirmed last on-treatment HIV-1 RNA $\geq 50$ copies/mL at premature discontinuation) and who had a VL $\geq 400$ copies/mL at failure (preferably confirmed, or at unconfirmed) or at later time points, including participants who discontinued with a last single VL $\geq 400$ copies/mL.

EMERALD included virologically suppressed adults, and therefore no standard resistance testing could be performed on screening or baseline samples. HIV-1 proviral DNA was analyzed retrospectively using GenoSure Archive ${ }^{\circledR}$ on baseline samples (VL $<50$ copies/mL) from participants with 
PDVR or prior VF (i.e., the participants most likely to have HIV-1 viruses with baseline resistance).

\section{Genotypic analysis}

Evaluation of screening and treatment-emergent RAMs (AMBER), or the presence of on-treatment (EMERALD) RAMs, was based on the PI, N(t)RTI, NNRTI, and integrase inhibitor International Antiviral Society [IAS]-USA mutation list. ${ }^{14}$ RAMs were considered developing (emerging) if they were detected postbaseline but not at screening.

\section{Phenotypic analysis}

Assessments of antiretroviral drug susceptibility (predicted phenotype, "sensitive," "resistance possible," or "resistant") were based on the genotype and applied algorithms (GenoSure MG or GenoSure Archive) or on the true phenotype, expressed as the fold change (FC) in the $50 \%$ effective concentration in cell-based assays $\left(\mathrm{EC}_{50}\right)$ below/equal or above, respectively, the lower clinical cutoff (when available) or biological cutoff (otherwise) for the PhenoSense GT report.

\section{Results}

\section{ART-naive participants (AMBER)}

Baseline characteristics. Overall, $18 \%$ of participants had HIV-1 VL $\geq 100,000$ copies/mL, and median baseline $\mathrm{CD} 4^{+}$count was 453 cells $/ \mathrm{mm} .{ }^{3,10}$ A majority of participants were infected with HIV-1 subtype B $(71 \%)$.
As depicted by the protocol at screening, all enrolled participants had HIV-1 viruses sensitive to DRV, emtricitabine, and tenofovir based on the genotype report. As expected in a treatment-naive population, few participants had viruses with $\geq 1$ primary PI RAMs ( $2 \%)$ or $\geq 1$ DRV RAMs (1\%) (Table 1). No participants had HIV-1 with $\geq 3$ DRV RAMs, which is correlated with DRV resistance. ${ }^{15}$ Secondary PI RAMs were observed in HIV-1 of almost all participants $(98 \%)$, reflecting the polymorphic nature of these mutations. NNRTI and N(t)RTI RAMs were detected in HIV1 of $16 \%$ and $5 \%$ of participants, respectively, with K103N and polymorphic E138E/A mutation being the most prevalent NNRTI RAMs (both 4\%) and A62A/V (3\%) the most prevalent N(t)RTI RAM (Table 1). No RAMs related to emtricitabine or tenofovir were detected.

Subgroup analyses showed that there was no effect of HIV1 subtype (B, non-B), presence of baseline primary PI and/or DRV RAMs, or N(t)RTI RAMs, NNRTI RAMs, M184I/V, and number of primary PI RAMs on virologic response at week 48 (FDA snapshot) (data not shown). Of the seven participants with viruses harboring a DRV RAM at screening (three in the D/C/F/TAF arm and four in the control arm), six had virologic response at week 48 and one discontinued due to other reasons with last available $\mathrm{VL}<50$ copies/mL (FDA snapshot).

Postbaseline resistance. Through 48 weeks, 8 (D/C/ F/TAF) and 6 (control) participants had PDVF, with virologic rebound occurring most frequently (Supplementary Fig. S1).

Table 1. Amber Genotype at Screening

\begin{tabular}{|c|c|c|c|}
\hline & $\begin{array}{c}D / C / F / T A F \\
800 / 150 / 200 / 10 m g \mathrm{~N}=362\end{array}$ & $\begin{array}{c}\text { Control regimen } \\
(D / C+F / T D F) \mathrm{N}=363\end{array}$ & $\begin{array}{c}\text { Total } \\
\mathrm{N}=725\end{array}$ \\
\hline Participants with genotype ${ }^{a}$ at screening & $N=361^{\mathrm{b}}$ & $N=362^{\mathrm{b}}$ & $N=723$ \\
\hline$\geq 1$ DRV RAM ${ }^{\mathrm{c}}, n(\%)$ & $3(1)$ & $4(1)$ & $7(1)$ \\
\hline $\mathrm{V} 11 \mathrm{~V} / \mathrm{A} / \mathrm{I} / \mathrm{T}$ & $3(1)$ & $3(1)$ & $6(1)$ \\
\hline $\mathrm{L} 33 \mathrm{~F}$ & 0 & $1(<1)$ & $1(<1)$ \\
\hline$\geq 1$ primary PI $\mathrm{RAM}^{\mathrm{c}}, n(\%)$ & $7(2)$ & $8(2)$ & $15(2)$ \\
\hline $\mathrm{M} 46 \mathrm{M} / \mathrm{I} / \mathrm{L}$ & 0 & $3(1)$ & $3(<1)$ \\
\hline Q58Q/E & $4(1)$ & $4(1)$ & $8(1)$ \\
\hline V82L & $1(<1)$ & 0 & $1(<1)$ \\
\hline L90M & $2(1)$ & $1(<1)$ & $3(<1)$ \\
\hline$\geq 1$ secondary PI RAM, $n(\%)$ & $354(98)$ & $354(98)$ & $708(98)$ \\
\hline$\geq 1 \mathrm{~N}(\mathrm{t}) \mathrm{RTI} \mathrm{RAM}^{\mathrm{c}}, n(\%)$ & $18(5)$ & $16(4)$ & $34(5)$ \\
\hline $\mathrm{M} 41 \mathrm{M} / \mathrm{L}$ & $3(1)$ & $2(1)$ & $5(1)$ \\
\hline $\mathrm{A} 62 \mathrm{~A} / \mathrm{V}$ & $10(3)$ & $11(3)$ & $21(3)$ \\
\hline D67N & $2(1)$ & $1(<1)$ & $3(<1)$ \\
\hline $\mathrm{K} 70 \mathrm{~K} / \mathrm{R}$ & 0 & $1(<1)$ & $1(<1)$ \\
\hline V75I & $1(<1)$ & 0 & $1(<1)$ \\
\hline L210W & $2(1)$ & $1(<1)$ & $3(<1)$ \\
\hline K219Q & $2(1)$ & $1(<1)$ & $3(<1)$ \\
\hline$\geq 1$ NNRTI RAM ${ }^{\mathrm{c}}, n(\%)$ & $55(15)$ & $63(17)$ & $118(16)$ \\
\hline V90I & $9(2)$ & $9(2)$ & $18(2)$ \\
\hline $\mathrm{K} 103 \mathrm{~N}$ & $12(3)$ & $14(4)$ & $26(4)$ \\
\hline V106I & $7(2)$ & $11(3)$ & $18(2)$ \\
\hline $\mathrm{E} 138 \mathrm{E} / \mathrm{A}$ & $12(3)$ & $16(4)$ & $28(4)$ \\
\hline V179D & $4(1)$ & $2(1)$ & $6(1)$ \\
\hline
\end{tabular}

${ }^{\mathrm{a}}$ GenoSure ${ }^{\circledR}$ MG.

${ }^{\mathrm{b}}$ One participant in each arm had failed screening genotypes and were enrolled based on local genotypes.

${ }^{\mathrm{c}}$ IAS-USA mutations ${ }^{16}$; all observed single and mixture of mutations were concatenated by RAM position.

D/C/F/TAF, darunavir/cobicistat/emtricitabine/tenofovir alafenamide once daily; Control regimen (D/C + F/TDF), darunavir/cobicistat plus emtricitabine/tenofovir disoproxil fumarate once daily; DRV, darunavir; RAM, resistance-associated mutation; PI, protease inhibitor; $\mathrm{N}(\mathrm{t}) \mathrm{RTI}$, nucleos(t)ide analog reverse transcriptase inhibitor; NNRTI, non-nucleoside analog reverse transcriptase inhibitor. 
Paired screening and postbaseline on-treatment genotypes were available for seven and two participants, respectively (Table 2).

No DRV, primary PI, or tenofovir RAMs emerged in HIV1 of any participant (Table 2).

The N(t)RTI RAM M184I/V, conferring phenotypic resistance to emtricitabine ( $\mathrm{FC}$ in $\left.\mathrm{EC}_{50}=56\right)$ and lamivudine $(\mathrm{FC}=143)$, but retaining sensitivity to abacavir, stavudine, tenofovir, and zidovudine, was identified at week 36 postPDVF by Sanger sequencing in only one participant receiving $\mathrm{D} / \mathrm{C} / \mathrm{F} / \mathrm{TAF}$. M184V was detected pretreatment at screening by deep sequencing as a minority variant (9\%). At week 12 only the wild-type genotype was detected, and at week 36, M184I (32\%) and M184V (67\%) were detected.

This participant had HIV-1 virus with transmitted NNRTI (efavirenz $[\mathrm{FC}=7] /$ nevirapine $[\mathrm{FC}=39]$ ) resistance due to the presence of $\mathrm{K} 103 \mathrm{~N}$ at screening. This participant was discontinued from the study due to noncompliance after the week 48 database lock. Further testing showed that the observed DRV plasma concentrations for this participant were low (ranging from 32.0 to $192 \mathrm{ng} / \mathrm{mL}$, except at week 4 [1,440 ng/mL]) and much lower than the anticipated DRV steady-state predose plasma concentration for this participant $(\sim 692 \mathrm{ng} / \mathrm{mL})$, which could be an indication that the participant did not take the medication regularly as recommended.

All other participants had virus that remained susceptible to all drugs in the treatment regimens based on genotypic/phenotypic assessments (PhenoSense GT assay).

One participant in the D/C/F/TAF arm had HIV-1 that developed the secondary PI RAM I62I/V. Three participants in the D/C/F/TAF arm and two participants in the control arm, none of whom met the definition of PDVF, had postbaseline off-treatment genotype data just after discontinuation (Table 2).
One of the three participants in the D/C/F/TAF arm had virus with an emerging secondary PI RAM I64I/L/M. As expected, these secondary PI RAMs had no effect on PI susceptibility.

\section{Virologically suppressed ART-experienced participants (EMERALD)}

Baseline characteristics. Most participants received DRV/ritonavir or DRV/cobicistat at screening. Median time since diagnosis was 9.26 years (interquartile range 4.22 18.12 years $).^{11}$

Of 1,141 participants, $664(58 \%)$ had received $\geq 5$ previous antiretrovirals (including screening ART and PI booster counted as a separate antiretroviral) and $312(27 \%)$ had received $\geq 8$ previous antiretrovirals; $472(41 \%)$ had received $\geq 2$ PIs, $474(42 \%) \geq 3$ N(t)RTIs, 340 (30\%) $\geq 1$ NNRTI, and $63(6 \%) \geq 1$ integrase inhibitor.

In total, $169(15 \%)$ participants had previous antiretroviral VF $(80$ [7\%] participants on a PI, 130 [11\%] on an N(t)RTI, 74 [6\%] on an NNRTI, and 10 [1\%] on an integrase inhibitor).

One participant who had HIV-1 with a V11I DRV RAM, which was a protocol deviation, reported on a historical genotype, was randomized and had a virologic response throughout the study until week 36 , after which the participant was lost to follow-up.

Archived baseline resistance. HIV-1 proviral DNA from baseline samples from all participants with prior $\mathrm{VF}$ $(N=169)$ or with PDVR $(N=27)$ (i.e., the subgroup most likely to have baseline resistance) was analyzed.

In the subgroup of all EMERALD participants with previous $\mathrm{VF}$ and genoarchive data $(N=140 ; 98 \mathrm{D} / \mathrm{C} / \mathrm{F} / \mathrm{TAF}$ and $42 \mathrm{bPI}+\mathrm{F} / \mathrm{TDF}), 4 \%(n=6 ; 4 \mathrm{D} / \mathrm{C} / \mathrm{F} / \mathrm{TAF}$ and $2 \mathrm{bPI}+$

Table 2. Amber and Emerald: Postbaseline Resistance Through Week 48

\begin{tabular}{|c|c|c|c|c|c|c|c|}
\hline \multirow[b]{2}{*}{ Study } & \multirow[b]{2}{*}{$A R T$} & \multirow[b]{2}{*}{$\begin{array}{l}\text { Participants, } \\
\mathrm{N}\end{array}$} & \multirow[b]{2}{*}{$\begin{array}{c}\text { Participants } \\
\text { with PDVF, } \\
\text { n (\%) }\end{array}$} & \multirow[b]{2}{*}{$\begin{array}{c}P D V F \\
\text { participants with } \\
\text { postbaseline } \\
\text { genotype } \\
\text { data, } \mathrm{n}(\%)\end{array}$} & \multirow[b]{2}{*}{$\begin{array}{c}\text { Non-PDVF } \\
\text { participants with } \\
\text { postbaseline } \\
\text { genotype } \\
\text { data, } \mathrm{n}(\%)\end{array}$} & \multicolumn{2}{|c|}{$\begin{aligned} \text { Participants with } & \geq 1 \\
\text { RAM }^{\mathrm{a}} \text { postbaseline } & , n(\%)\end{aligned}$} \\
\hline & & & & & & $\begin{array}{c}\text { Reverse } \\
\text { transcriptase } \\
\text { FTC } \\
\text { or TFV }\end{array}$ & $\begin{array}{c}\text { Protease } \\
\text { Primary } \\
\text { PI or DRV }\end{array}$ \\
\hline \multirow[t]{2}{*}{ AMBER } & D/C/F/TAF & 362 & $8(2)$ & $7(2)$ & $3(1)$ & $\mathrm{M} 184 \mathrm{I} / \mathrm{V}^{\mathrm{c}} ; n=1$ & $\mathbf{0}$ \\
\hline & $\begin{array}{l}\text { Control regimen } \\
(\mathrm{D} / \mathrm{C}+\mathrm{F} / \mathrm{TDF})\end{array}$ & 363 & $6(2)$ & $2(1)$ & $2(1)$ & 0 ' & 0 \\
\hline \multirow[t]{2}{*}{ EMERALD } & $\mathrm{D} / \mathrm{C} / \mathrm{F} / \mathrm{TAF}$ & 763 & $19(2)$ & $\mathbf{1}^{\mathrm{e}}(<\mathbf{1})$ & $2^{\mathrm{f}}(<1)$ & 0 & 0 \\
\hline & $\begin{array}{l}\text { Control regimen } \\
\left.\text { (bPI }^{\mathrm{d}}+\mathrm{F} / \mathrm{TDF}\right)\end{array}$ & 378 & $8(2)$ & $3^{g}(1)$ & 0 & 0 & 0 \\
\hline Total & D/C/F/TAF & 1125 & $27(2)$ & $8(1)$ & $5(<1)$ & $1(<1)$ & $\mathbf{0}$ \\
\hline
\end{tabular}

All data from the DCFTAF groups are bolded.

${ }^{a}$ IAS-USA 2015 mutation list ${ }^{16}$; all observed single and mixture of mutations were concatenated by RAM position.

${ }^{\mathrm{b}}$ At unconfirmed or confirmed virologic failure or later time point(s).

${ }^{\mathrm{c}}$ Conferring phenotypic resistance to emtricitabine and lamivudine, but retaining sensitivity to abacavir, tenofovir, stavudine, and zidovudine; this participant had HIV-1 virus with transmitted efavirenz and nevirapine resistance shown by the presence of K103N at screening; MI84V was detected by deep sequencing (9\%) at screening.

${ }^{\mathrm{d}}$ At screening, 266 participants were on boosted DRV ( $n=202$ DRV/ritonavir; $n=64$ DRV/cobicistat), 82 on boosted atazanavir $(n=81$ atazanavir/ritonavir; $n=1$ atazanavir/cobicistat), and 30 on lopinavir/ritonavir.

${ }^{\mathrm{e}}$ One rebounder in the D/C/F/TAF arm had an N(t)RTI thymidine analog-associated mutation (TAM), D67D/N.

${ }^{\mathrm{f}}$ One of these participants had a K103K/N NNRTI RAM conferring resistance to efavirenz and nevirapine.

${ }^{\mathrm{g}}$ One rebounder in the control arm had an NNRTI RAM, E138E/G, conferring resistance to rilpivirine; this mutation was not related to any of the study drugs and was probably related to previous ART use.

ART, antiretroviral treatment; PDVF, protocol-defined virologic failure; RAM, resistance-associated mutation; FTC, emtricitabine; TFV, tenofovir; bPI, boosted protease inhibitor; DRV, darunavir; D/C/F/TAF, darunavir/cobicistat/emtricitabine/tenofovir alafenamide; F/TDF, emtricitabine/tenofovir disoproxil fumarate. 
Table 3. Emerald: Prevalence of Baseline RAMS in HIV-1 Proviral DNA for Participants with Previous Virologic Failure

\begin{tabular}{|c|c|c|c|}
\hline & $\begin{array}{c}D / C / F / T A F \\
800 / 150 / 200 / 10 m g \quad \mathrm{~N}=763\end{array}$ & $\begin{array}{c}\text { Control regimen } \\
(b P I+F / T D F) \mathrm{N}=378\end{array}$ & Total $\mathrm{N}=1,141$ \\
\hline Participants with previous VF, $N$ & 116 & 53 & 169 \\
\hline $\begin{array}{l}\text { Participants with previous VF and } \\
\text { genoarchive }^{\mathrm{a}} \text { data at baseline, } N^{\mathrm{b}}\end{array}$ & 98 & 42 & 140 \\
\hline \multicolumn{4}{|l|}{ Genotypic Susceptibility, $n(\%)$ : } \\
\hline All PIs & $80(82)$ & $36(86)$ & $116(83)$ \\
\hline Boosted ATV & $91(93)$ & $40(95)$ & $131(94)$ \\
\hline Boosted DRV & $98(100)$ & $42(100)$ & $140(100)$ \\
\hline Boosted LPV & $94(96)$ & $40(95)$ & $134(96)$ \\
\hline All N(t)RTIs & $53(54)$ & $19(45)$ & $72(51)$ \\
\hline FTC & $62(63)$ & $21(50)$ & $83(59)$ \\
\hline TFV & $76(78)$ & $30(71)$ & $106(76)$ \\
\hline All NNRTIs & $56(57)$ & $29(69)$ & $85(61)$ \\
\hline All INIs & $86(88)$ & $39(93)$ & $125(89)$ \\
\hline DTG & $97(99)^{\mathrm{c}}$ & $41(98)^{c}$ & $138(99)$ \\
\hline EVG & $89(91)$ & $40(95)$ & $129(92)$ \\
\hline RTG & $86(88)$ & $39(93)$ & $125(89)$ \\
\hline$\geq 1$ DRV RAMs ${ }^{\mathrm{d}}, n(\%)$ & $4^{e}(4)$ & $2(5)$ & $6(4)$ \\
\hline $\mathrm{L} 33 \mathrm{~L} / \mathrm{F}$ & 0 & $1(2)$ & $1(1)$ \\
\hline $\mathrm{T} 74 \mathrm{~T} / \mathrm{P}$ & 0 & $1(2)$ & $1(1)$ \\
\hline $\mathrm{L} 76 \mathrm{~L} / \mathrm{V}$ & $1(1)$ & 0 & $1(1)$ \\
\hline $\mathrm{I} 84 \mathrm{I} / \mathrm{V}$ & $4(4)$ & 0 & $4(3)$ \\
\hline$\geq 1$ primary PI RAMs ${ }^{\mathrm{d}}, n(\%)$ & $20(20)$ & 7 (17) & 27 (19) \\
\hline $\mathrm{D} 30 \mathrm{D} / \mathrm{N}$ & $4(4)$ & 0 & $4(3)$ \\
\hline $\mathrm{M} 46 \mathrm{M} / \mathrm{I} / \mathrm{L}$ & $8(8)$ & $5(12)$ & $13(9)$ \\
\hline $\mathrm{I} 50 \mathrm{I} / \mathrm{L}$ & 0 & $1(2)$ & $1(1)$ \\
\hline $\mathrm{Q} 58 \mathrm{Q} / \mathrm{E}$ & 0 & $1(2)$ & $1(1)$ \\
\hline $\mathrm{T} 74 \mathrm{~T} / \mathrm{P}$ & 0 & $1(2)$ & $1(1)$ \\
\hline L76L/V & $1(1)$ & 0 & $1(1)$ \\
\hline V82V/A/T & $1(1)$ & $2(5)$ & $3(2)$ \\
\hline I84I/V & $4(4)$ & 0 & $4(3)$ \\
\hline N88N/S & $2(2)$ & 0 & $2(1)$ \\
\hline L90L/I/M & $8(8)$ & $3(7)$ & $11(8)$ \\
\hline$\geq 1 \mathrm{~N}(\mathrm{t}) \mathrm{RTI}$ RAMs, $n(\%)$ & $46(47)$ & $23(55)$ & $69(49)$ \\
\hline$\geq 1$ tenofovir RAMs ${ }^{\mathrm{d}}, n(\%)$ & $4(4)$ & $1(2)$ & $5(4)$ \\
\hline $\mathrm{K} 65 \mathrm{~K} / \mathrm{R}$ & $4(4)$ & 0 & $4(3)$ \\
\hline $\mathrm{K} 70 \mathrm{~K} / \mathrm{D} / \mathrm{E} / \mathrm{N}$ & 0 & $1(2)$ & $1(1)$ \\
\hline$\geq 1 \mathrm{TAMs}^{\mathrm{d}, \mathrm{f}} n(\%)$ & $34(35)$ & $14(33)$ & $48(34)$ \\
\hline $\mathrm{M} 41 \mathrm{M} / \mathrm{L}$ & $18(18)$ & 8 (19) & $26(19)$ \\
\hline $\mathrm{D} 67 \mathrm{D} / \mathrm{N}$ & $10(10)$ & 7 (17) & $17(12)$ \\
\hline $\mathrm{K} 70 \mathrm{~K} / \mathrm{R}$ & $18(18)$ & $6(14)$ & $24(17)$ \\
\hline L210L/W & $9(9)$ & $3(7)$ & $12(9)$ \\
\hline T215A/C/D/E/F/G/I/L/N/S/T/Y & $20(20)$ & $12(29)$ & $32(23)$ \\
\hline $\mathrm{K} 219 \mathrm{~K} / \mathrm{E} / \mathrm{Q}$ & $11(11)$ & 4 (10) & $15(11)$ \\
\hline$\geq 1$ emtricitabine $\mathrm{RAMs}^{\mathrm{d}}, n(\%)$ & $35(36)$ & $18(43)$ & $53(38)$ \\
\hline $\mathrm{K} 65 \mathrm{~K} / \mathrm{R}$ & $4(4)$ & 0 & $4(3)$ \\
\hline $\mathrm{M} 184 \mathrm{M} / \mathrm{I} / \mathrm{V}$ & $31(32)$ & $18(43)$ & $49(35)$ \\
\hline$\geq 1$ NNRTI RAMs, $n(\%)$ & $44(45)$ & $14(33)$ & $58(41)$ \\
\hline V90V/I & $5(5)$ & $3(7)$ & $8(6)$ \\
\hline A98A/G/S & $5(5)$ & $1(2)$ & $6(4)$ \\
\hline $\mathrm{L} 100 \mathrm{~L} / \mathrm{I}$ & $2(2)$ & 0 & $2(1)$ \\
\hline $\mathrm{K} 101 \mathrm{~K} / \mathrm{E} / \mathrm{P} / \mathrm{Q} / \mathrm{T}$ & $7(7)$ & $1(2)$ & $8(6)$ \\
\hline $\mathrm{K} 103 \mathrm{~K} / \mathrm{N} / \mathrm{R} / \mathrm{S}$ & 19 (19) & $6(14)$ & $25(18)$ \\
\hline V106V/A/I & $3(3)$ & $1(2)$ & $4(3)$ \\
\hline $\mathrm{V} 108 \mathrm{~V} / \mathrm{I} / \mathrm{M}$ & $6(6)$ & $1(2)$ & $7(5)$ \\
\hline $\mathrm{E} 138 \mathrm{E} / \mathrm{A} / \mathrm{G} / \mathrm{K} / \mathrm{P} / \mathrm{Q} / \mathrm{R}$ & $13(13)$ & $1(2)$ & $14(10)$ \\
\hline $\mathrm{V} 179 \mathrm{~V} / \mathrm{A} / \mathrm{F} / \mathrm{I} / \mathrm{T}$ & $2(2)$ & 0 & $2(1)$ \\
\hline Y181Y/C & $8(8)$ & $3(7)$ & $11(8)$ \\
\hline Y188Y/H/L & $3(3)$ & 0 & $3(2)$ \\
\hline
\end{tabular}


TABle 3. (CONTINUED)

\begin{tabular}{|c|c|c|c|}
\hline & $\begin{array}{c}\text { D/C/F/TAF } \\
800 / 150 / 200 / 10 m g \mathrm{~N}=763\end{array}$ & $\begin{array}{c}\text { Control regimen } \\
(b P I+F / T D F) \mathrm{N}=378\end{array}$ & Total $\mathrm{N}=1,141$ \\
\hline G190G/A/R/S & $8(8)$ & $4(10)$ & $12(9)$ \\
\hline $\mathrm{H} 221 \mathrm{H} / \mathrm{Y}$ & $1(1)$ & $1(2)$ & $2(1)$ \\
\hline $\mathrm{P} 225 \mathrm{P} / \mathrm{H}$ & $1(1)$ & $1(2)$ & $2(1)$ \\
\hline $\mathrm{M} 230 \mathrm{M} / \mathrm{L}$ & 0 & $1(2)$ & $1(1)$ \\
\hline$\geq 1$ Primary INI RAMs, $n(\%)$ & $5(5)$ & $1(2)$ & $6(4)$ \\
\hline $\mathrm{T} 66 \mathrm{~T} / \mathrm{I}$ & $2(2)$ & 0 & $2(1)$ \\
\hline $\mathrm{Q} 148 \mathrm{Q} / \mathrm{H} / \mathrm{R}$ & $1(1)$ & $1(2)$ & $2(1)$ \\
\hline $\mathrm{N} 155 \mathrm{~N} / \mathrm{H}$ & $2(2)$ & 0 & $2(1)$ \\
\hline
\end{tabular}

${ }^{\mathrm{a}}$ GenoSure Archive ${ }^{\circledR}$.

${ }^{\mathrm{b}}$ Denominator for the prevalence of baseline RAMs. ${ }^{16}$

${ }^{\text {c }}$ Two participants showed possible resistance to DTG and full resistance to RTG and EVG (integrase inhibitor RAMs: G140S, Q148H and G140A, Q148R) on the genotype report. Both participants had previously virologically failed on RTG.

${ }^{\mathrm{d}}$ Observed single and mixture of mutations were concatenated by RAM position.

${ }^{\mathrm{e}}$ In one participant, 2 DRV RAMs were observed (I84I/V and L76L/V).

${ }^{f}$ Thymidine analog-associated mutations (TAMs): M41L, D67N, K70R, L210W, T215Y/F, K291Q/E. ${ }^{16,17}$

Tenofovir resistance is associated with the presence of $\geq 3$ TAMs, inclusive of either M41L or L210W.

$\mathrm{D} / \mathrm{C} / \mathrm{F} / \mathrm{TAF}$, darunavir/cobicistat/emtricitabine/tenofovir alafenamide once daily; Control regimen (bPI + F/TDF), boosted protease inhibitor plus emtricitabine/tenofovir disoproxil fumarate once daily (At screening, 266 participants were on boosted DRV $[n=202$ DRV/ritonavir; $n=64 \mathrm{DRV} /$ cobicistat], 82 on boosted ATV [ $n=81 \mathrm{ATV} /$ ritonavir; $n=1 \mathrm{ATV} /$ cobicistat], and 30 on LPV/ritonavir)

VF, virologic failure; PI, protease inhibitor; ATV, atazanavir; DRV, darunavir; LPV, lopinavir; N(t)RTI, nucleos(t)ide analog reverse transcriptase inhibitor; FTC, emtricitabine; TFV, tenofovir; NNRTI, non-nucleoside analogue reverse transcriptase inhibitor; INI, integrase inhibitor; DTG, dolutegravir; EVG, elvitegravir; RTG, Raltegravir; RAM, resistance-associated mutation.

F/TDF) had viruses with DRV RAMs, 4\% with tenofovir RAMs $(n=5)$ (Table 3$)$, and $21 \%$ with $\geq 3$ thymidine analogassociated mutations (TAMs) $(n=29)$. Emtricitabine RAMs were seen in $38 \%(n=53)$, mainly at RT position $184(35 \%)$ (Table 3 ). Predicted phenotype showed that $100 \%$ of participants had HIV-1 susceptible to DRV, 59\% to emtricitabine, and $76 \%$ to tenofovir; $83 \%$ to all PIs, $51 \%$ to all N(t)RTIs, and $61 \%$ to all NNRTIs (Table 3 ). All these participants with virus harboring archived DRV, emtricitabine, and tenofovir resistance achieved virologic response (FDA snapshot) at week 48 or at last on-treatment VL.

In the subgroup of participants with PDVR and genoarchive data $(n=24)$, none had HIV-1 with archived RAMs to
DRV, emtricitabine, or tenofovir (Table 4). Also no primary PI RAMs nor TAMs were observed.

Postbaseline resistance. In EMERALD, as there were few participants with PDVR throughout 48 weeks $(19 / 763$ D/C/F/TAF arm and 8/378 control arm), few samples were eligible for postbaseline genotyping (participants whose VL rebounded with $\mathrm{VL} \geq 400$ copies/mL at failure or at later time points or at discontinuation). Only three participants in the $\mathrm{D} / \mathrm{C} / \mathrm{F} / \mathrm{TAF}$ arm and zero participants in the control arm had virologic rebound using the $\mathrm{VL} \geq 200$ copies/mL threshold. One participant whose VL rebounded in the $\mathrm{D} / \mathrm{C} / \mathrm{F} / \mathrm{TAF}$ arm and three participants that rebounded in the

Table 4. Emerald: Prevalence of Baseline RaMS in HiV-1 Proviral DNA for Participants with PDVR

\begin{tabular}{|c|c|c|c|}
\hline & $\begin{array}{c}D / C / F / T A F \\
800 / 150 / 200 / 10 m g \mathrm{~N}=763\end{array}$ & $\begin{array}{c}\text { Control regimen } \\
(b P I+F / T D F) \mathrm{N}=378\end{array}$ & $\begin{array}{c}\text { Total } \\
\mathrm{N}=1,141\end{array}$ \\
\hline Participants with PDVR, $N$ & 19 & 8 & 27 \\
\hline $\begin{array}{l}\text { Participants with PDVR and genoarchive } \\
\text { data at baseline, } N^{\mathrm{b}}\end{array}$ & 17 & 7 & 24 \\
\hline \multicolumn{4}{|l|}{ Genotypic Susceptibility, $n(\%)$} \\
\hline All PIs & $16^{\mathrm{c}}(94)$ & $7(100)$ & $23^{\mathrm{c}}(96)$ \\
\hline All N(t)RTIs & 17 (100) & 7 (100) & $24(100)$ \\
\hline All NNRTIs & $13(76)$ & 7 (100) & $20(83)$ \\
\hline All INIs & $17(100)$ & $7(100)$ & $24(100)$ \\
\hline$\geq 1$ DRV RAMs/primary PI RAMs, $n(\%)$ & 0 & 0 & 0 \\
\hline$\geq 1$ tenofovir RAMs, $n(\%)$ & 0 & 0 & 0 \\
\hline$\geq 1$ TAMs $n(\%)$ & 0 & 0 & 0 \\
\hline$\geq 1$ emtricitabine RAMs, $n(\%)$ & 0 & 0 & 0 \\
\hline
\end{tabular}

${ }^{\mathrm{a}}$ GenoSure Archive.

${ }^{\mathrm{b}}$ Denominator for the prevalence of baseline RAMs. ${ }^{16}$

${ }^{c}$ One participant had HIV-1 virus not susceptible to nelfinavir or unboosted atazanavir.

$\mathrm{D} / \mathrm{C} / \mathrm{F} / \mathrm{TAF}$, darunavir/cobicistat/emtricitabine/tenofovir alafenamide once daily; Control regimen (bPI + F/TDF), boosted protease inhibitor plus emtricitabine/tenofovir disoproxil fumarate once daily (At screening, 266 participants were on boosted DRV $[n=202$ DRV/ritonavir; $n=64 \mathrm{DRV/cobicistat],} 82$ on boosted atazanavir [ $n=81$ atazanavir/ritonavir; $n=1$ atazanavir/cobicistat], and 30 on lopinavir/ritonavir); PDVR, protocol-defined virologic rebound; PI, protease inhibitor; $\mathrm{N}(\mathrm{t}) \mathrm{RTI}$, nucleos(t)ide analog reverse transcriptase inhibitor; NNRTI, non-nucleoside analogue reverse transcriptase inhibitor; INI, integrase inhibitor; DRV, darunavir; RAM, resistanceassociated mutation; TAM, thymidine analog-associated mutation. 
control arm had postbaseline genotypes (Table 2 and Supplementary Fig. S2).

For the four postbaseline PR and RT sequences, the predicted phenotypic assessment (based on the GenoSure MG assay) was that all participants had HIV-1 virus susceptible to all the drugs in the treatment regimen and no DRV, primary PI, tenofovir, or emtricitabine RAMs were observed (Table 2).

One participant with PDVR in the D/C/F/TAF arm had virus with one TAM, D67D/N, and one participant with PDVR in the control arm had HIV-1 with an NNRTI RAM, E138E/G, conferring resistance to rilpivirine. Two further participants in the D/C/F/TAF arm who discontinued at week 12, but whose VL did not rebound (assessed as "no VL data in the week 48 window" by the FDA-snapshot analysis), had a genotype at a posttreatment follow-up visit. One of these participants had virus with a K103K/N NNRTI RAM conferring resistance to efavirenz, which was probably related to previous use of efavirenz, emtricitabine, and TDF (Atripla).

\section{Discussion}

The week 48 analyses of the two investigational Phase III, randomized, controlled trials showed that the D/C/F/TAF once-daily STR had a response rate of $91 \%$ in ART-naive participants in AMBER and 95\% in virologically suppressed participants in EMERALD, with VF rates of $4 \%$ and $1 \%$, respectively, and a high genetic barrier to resistance. Through week 48 in both studies, no treatment-emergent DRV or primary PI RAMs were observed in the HIV-1 of 1,125 participants receiving $\mathrm{D} / \mathrm{C} / \mathrm{F} / \mathrm{TAF}$, or in 629 participants receiving boosted DRV in combination with F/TDF in the control arms. No tenofovir RAM or tenofovir resistance was observed in either trial. The most common N(t)RTI RAM, $\mathrm{M} 184 \mathrm{I} / \mathrm{V}$ (conferring high-level in vitro resistance to emtricitabine and lamivudine), was identified post-VF in HIV-1 of only one participant in the D/C/F/TAF arm of AMBER. However, the presence of $\mathrm{M} 184 \mathrm{I} / \mathrm{V}$ is not a contraindication to the use of emtricitabine and lamivudine, because these RAMs increase tenofovir susceptibility and decrease viral replication fitness. ${ }^{18}$ For this participant, M184V was detected in the virus pretreatment by deep sequencing (Illumina MiSeq) as a minority variant (9\%). This participant also had HIV-1 with transmitted NNRTI (efavirenz/nevirapine) resistance as shown by the presence of K103N at screening. This participant was potentially nonadherent as indicated by the low DRV plasma concentrations, which were lower than the steady-state predose concentration, and the participant discontinued from the study due to noncompliance.

These results are consistent with those of a previous metaanalysis of postbaseline resistance among participants in the DRV $800 \mathrm{mg}$ once-daily dosing arms from seven clinical Phase II and III studies. ${ }^{9}$ Only four of 1,686 (0.2\%) participants had viruses that developed postbaseline primary PI and/or DRV RAMs, and only one of 1,686 $(<0.1 \%)$ participant had HIV-1 that lost DRV phenotypic susceptibility after having previously failed lopinavir/ritonavir. To our knowledge, no participant that has not previously failed another PI has ever failed with HIV-1 that was phenotypically resistant to DRV. Only 10 of 1,103 participants from the seven clinical trials using an $\mathrm{N}(\mathrm{t}) \mathrm{RTI}$ backbone $(0.9 \%)$ had viruses that developed $\geq 1 \mathrm{~N}(\mathrm{t}) \mathrm{RTI}$ RAM, and eight of those had HIV-1 harboring the emtricitabine RAM M184I/V; no tenofovir RAM or tenofovir resistance was detected. Other studies investigating F/TAF containing STR regimens showed similar results. Two Phase III studies investigating virologically suppressed participants who switched to rilpivirine/emtricitabine/tenofovir alafenamide reported zero and two M184V RAMs postbaseline out of $438^{19}$ and $316^{20}$ participants, respectively. Of 959 virologically suppressed participants assigned to the elvitegravir/cobicistat/emtricitabine/ tenofovir (E/C/F/TAF) alafenamide arm, one had HIV-1 that developed M184M/I. ${ }^{21}$ In two Phase III studies in ART-naive adults investigating the E/C/F/TAF STR (in total 866 participants were in the E/C/F/TAF arm), development of the M184V/I RAM was observed in HIV-1 of $11 \mathrm{E} / \mathrm{C} / \mathrm{F} / \mathrm{TAF}$ participants and the K65R/N RAM in viruses of two participants. $^{22,23}$ In Phase III studies of bictegravir, emtricitabine, and tenofovir alafenamide (B/F/TAF) in ART-naive ${ }^{24,25}$ and virologically suppressed adults, ${ }^{26,27}$ no treatment emergent resistance to the components of the treatment regimen were identified.

In AMBER through week 48, only eight out of 362 ARTnaive participants in the D/C/F/TAF arm and six out of 363 in the control arm had PDVF, with paired screening and postbaseline on-treatment genotypes available for seven and two participants, respectively. ${ }^{10}$ Similarly, as there were few participants whose VL rebounded through 48 weeks in EMERALD, with most participants having low VL values, samples from only one participant who rebounded in the $\mathrm{D} / \mathrm{C} / \mathrm{F} / \mathrm{TAF}$ arm, and three who rebounded in the control arm were eligible for post-week $48 \mathrm{HIV}-1$ genotypic resistance analysis. Most participants with rebound had resuppressed VL by week 48 and confirmed rebounds $\geq 200$ copies $/ \mathrm{mL}$ occurred in only three participants in the D/C/F/TAF arm and none in the control arm. ${ }^{11}$

In EMERALD, exclusion criteria were less restrictive (and thus participants may be more representative of the real world) than in other recent switch studies for HIV ART. ${ }^{26-33}$ While participants in EMERALD were ART experienced, with $58 \%$ of participants having received $\geq 5$ previous antiretroviral agents, and $15 \%$ had previous VF, few participants had PDVR cumulative through week 48. Subgroup analyses by previous antiretroviral use and VF showed no effect on rebound rates, virologic response, and VF rates by the FDAsnapshot analysis. ${ }^{11,34}$ There was no exclusion on the basis of tenofovir or emtricitabine RAMs. In EMERALD D/C/F/TAF participants with previous VF $(n=98)$ at baseline, $37 \%$ $(n=36)$ had HIV-1 not fully susceptible to emtricitabine and $22 \%(n=22)$ not fully susceptible to tenofovir (based on the genoarchive assay); baseline archived RAMs to tenofovir (4 D/C/F/TAF participants) and emtricitabine (35 D/C/F/ TAF participants, mainly 31 with $\mathrm{M} 184 \mathrm{I} / \mathrm{V}$ ) were observed. Nineteen percent $(n=19)$ had HIV-1 with $\geq 3$ TAMs, of which $9 \%(n=9)$ had virus with L210W and $18 \%(n=18)$ with M41L (tenofovir resistance is also associated with the presence of $\geq 3$ TAMs, inclusive of either M41L or L210W). ${ }^{17}$ This observed archived resistance did not preclude virologic response in $\mathrm{D} / \mathrm{C} / \mathrm{F} / \mathrm{TAF}$ participants. In participants with PDVR, none had viruses with archived RAMs to DRV, emtricitabine, and tenofovir. The clinical utility of proviral DNA resistance has yet to be fully established. These nextgeneration sequencing genotypic assays that analyze HIV-1 proviral DNA might miss some previous drug resistance 
mutations found in plasma viral RNA and vice versa, and they should be interpreted with caution. ${ }^{1,35,36}$

In EMERALD, the only exclusion criterion for presence of mutations was the presence of $\geq 1$ DRV RAMs if historical genotypes were available (or if not, previous VF on DRV-based regimens). This exclusion criterion was introduced in line with the DRV once-daily indication in ART-experienced virologically failing participants. Efficacy from a pooled dataset of studies in highly ART-experienced participants, who had received treatment with DRV/ritonavir 600/100 mg twice daily, was shown to be compromised only in the presence of $\geq 3 \mathrm{DRV}$ RAMs in the background of a high number of PI RAMs (median of 14 or 15 , respectively). ${ }^{15}$ There were three participants with HIV-1 harboring a DRV RAM (V11I) pre-D/C/F/TAF treatment in AMBER, all who had virologic response at week 48 (or at discontinuation) by the FDA-snapshot analysis. These observations are also in line with Lathouwers et al. ${ }^{9}$ who showed that of 14 participants who had viruses with a DRV RAM at baseline and received DRV $800 \mathrm{mg}$ once daily-based regimens, all achieved virologic response. In EMERALD, in the subgroup with previous VF or PDVR receiving D/C/F/TAF with genoarchive data, 4 (all previous VFs) participants had HIV-1 with archived DRV RAMs (all had viruses harboring one DRV RAM and one participant had virus with 2 DRV RAMs) at baseline. All of these 4 participants had virologic response at week 48 (FDA-snapshot analysis).

$\mathrm{D} / \mathrm{C} / \mathrm{F} / \mathrm{TAF}$ may have an important role for treating patients with uncertain adherence or for rapid initiation in patients for whom resistance testing results are not yet available. ${ }^{1,3}$ Furthermore, D/C/F/TAF does not require HLA $\mathrm{B} * 5701$ screening or hepatitis testing before treatment initiation. All these characteristics suggest D/C/F/TAF is a feasible option in a test and treat setting or for ART-naive patients, where rapid treatment is justified. D/C/F/TAF is being evaluated in the first known Phase III trial (single arm; $N=109$ ) of an STR in a rapid initiation model in the DIAMOND study (NCT03227861). ${ }^{37}$ In the primary intent-totreat analysis, $84 \%$ of patients (92/109) achieved virologic response at week 48 (FDA-snapshot analysis).

In conclusion, $\mathrm{D} / \mathrm{C} / \mathrm{F} / \mathrm{TAF}$ has a high genetic barrier to resistance in ART-naive and ART-experienced adults living with HIV-1, even in those with virus harboring archived study drug RAMs at baseline. The D/C/F/TAF STR combines the efficacy and high genetic barrier to resistance of DRV with the safety advantages of TAF to provide a new option for people living with HIV-1.

\section{Acknowledgments}

The authors thank the participants and their families for their participation and support during the studies, the central and local Janssen AMBER and EMERALD study teams, study center staff, and the principal investigators.

They also thank other Janssen staff members for their input into this article. They also acknowledge Ian Woolveridge (Zoetic Science, an Ashfield company, Macclesfield, UK) for assistance in drafting the article and coordinating and collating author contributions, which was funded by Janssen.

\section{AMBER Principal Investigators}

Belgium: S De Wit, E Florence, L Vandekerckhove, B Vandercam; Canada: J Brunetta, M Klein, D Murphy, A
Rachlis, S Walmsley; France: F Ajana, L Cotte, P-M Girard, C Katlama, J-M Molina, I Poizot-Martin, F Raffi, D Rey, J Reynes, E Teicher, and Y Yazdanpanah; Germany: K Arastéh, M Bickel, J Bogner, S Esser, G Faetkenheuer, H Jessen, W Kern, J Rockstroh, C Spinner, H-J Stellbrink, and A Stoehr; Italy: A Antinori, F Castelli, A Chirianni, A De Luca, A Di Biagio, M Galli, A Lazzarin, F Maggiolo, R Maserati, and C Mussini; Poland: A Garlicki, J Gasiorowski, W Halota, A Horban, M Parczewski, and A Piekarska; Russia: E Belonosova, O Chernova, N Dushkina, V Kulagin, E Ryamova, A Shuldyakov, N Sizova, O Tsybakova, E Voronin, and A Yakovlev; Spain: A Antela, JR Arribas, J Berenguer, J Casado, V Estrada, MJ Galindo, M Garcia Del Toro, JM Gatell, M Gorgolas, F Gutierrez, MDM Gutierrez, E Negredo, JA Pineda, D Podzamczer, J Portilla Sogorb, A Rivero, R Rubio, P Viciana, and I De Los Santos; UK: A Clarke, BG Gazzard, MA Johnson, C Orkin, I Reeves, and L Waters; USA: P Benson, L Bhatti, F Bredeek, G Crofoot, D Cunningham, E DeJesus, J Eron, F Felizarta, R Franco, J Gallant, D Hagins, K Henry, D Jayaweera, C Lucasti, C Martorell, C McDonald, J McGowan, A Mills, J Morales-Ramirez, D Prelutsky, M Ramgopal, B Rashbaum, P Ruane, J Slim, A Wilkin, and J deVente.

\section{EMERALD Principal Investigators}

Belgium: S De Wit, E Florence, M Moutschen, E Van Wijngaerden, L Vandekerckhove, and B Vandercam; Canada: J Brunetta, B Conway, M Klein, D Murphy, A Rachlis, S Shafran, and S Walmsley; France: F Ajana, L Cotte, P-M Girard, C Katlama, J-M Molina, I Poizot-Martin, F Raffi, D Rey, J Reynes, E Teicher, and Y Yazdanpanah; Poland: J Gasiorowski, W Halota, A Horban, A Piekarska, and A Witor; Spain: JR Arribas, I Perez-Valero, J Berenguer, J Casado, JM Gatell, F Gutierrez, MJ Galindo, MDM Gutierrez, JA Iribarren, H Knobel, E Negredo, JA Pineda, D Podzamczer, J Portilla Sogorb, F Pulido, C Ricart, A Rivero, I Santos Gil; Sweden: A Blaxhult, L Flamholc, M Gisslèn, and A Thalme; Switzerland: J Fehr, A Rauch, and M Stoeckle; UK: A Clarke, BG Gazzard, MA Johnson, C Orkin, F Post, A Ustianowski, and L Waters; USA: J Bailey, P Benson, L Bhatti, I Brar, UF Bredeek, C Brinson, G Crofoot, D Cunningham, E DeJesus, C Dietz, R Dretler, J Eron, F Felizarta, C Fichtenbaum, J Gallant, J Gathe, D Hagins, S Henn, WK Henry, G Huhn, M Jain, C Lucasti, C Martorell, C McDonald, A Mills, J Morales-Ramirez, K Mounzer, R Nahass, H Olivet, O Osiyemi, D Prelutsky, M Ramgopal, B Rashbaum, G Richmond, P Ruane, A Scarsella, A Scribner, P Shalit, D Shamblaw, J Slim, K Tashima, G Voskuhl, D Ward, A Wilkin, and J de Vente.

\section{Author Disclosure Statement}

Janssen sponsored the studies. E.L., E.Y.W., K.B., B.B., A.G., J.J., E.M., E.V.L., M.O., and S.D.M. are all full-time employees of Janssen and potential stockholders of Johnson \& Johnson. The data sharing policy of Janssen Pharmaceutical Companies of Johnson \& Johnson is available at www.janssen .com/clinical-trials/transparency. As noted on this site, requests for access to the study data can be submitted through Yale Open Data Access (YODA) Project site at http://yoda .yale.edu.

E.L., E.Y.W., K.B., B.B., A.G., J.J., E.M., E.V.L., M.O., and S.D.M. were involved in the data analyses. All authors 
were involved in the development of the primary article, interpretation of data, and have read and approved the final version, and have met the criteria for authorship as established by the ICMJE.

\section{Funding Information}

This study was sponsored by Janssen.

\section{Supplementary Material}

Supplementary Figure S1

Supplementary Figure S2

\section{References}

1. Panel on Antiretroviral Guidelines for Adults and Adolescents: Guidelines for the Use of Antiretroviral Agents in Adults and Adolescents Living with HIV. Department of Health and Human Services (Updated July 10, 2019). Available at www.aidsinfo.nih.gov/ContentFiles/Adultand AdolescentGL.pdf, accessed October 7, 2019

2. EACS. European AIDS Clinical Society Guidelines, Version 9.1: (October 2018). Available at www.eacsociety.org/ files/2018_guidelines-9.1-english.pdf, accessed October 7, 2019

3. Saag MS, Benson CA, Gandhi RT, et al.: Antiretroviral drugs for treatment and prevention of HIV infection in adults: 2018 recommendations of the International Antiviral Society-USA Panel. JAMA 2018;320:379-396.

4. Orkin C, DeJesus E, Khanlou H, et al.: Final 192-week efficacy and safety of once-daily darunavir/ritonavir compared with lopinavir/ritonavir in HIV-1-infected treatmentnaïve patients in the ARTEMIS trial. HIV Med 2013;14: 49-59.

5. Cahn P, Fourie J, Grinsztejn B, et al.: Week 48 analysis of once-daily vs. twice-daily darunavir/ritonavir in treatmentexperienced HIV-1-infected patients. AIDS 2011;25:929939.

6. Flynn P, Komar S, Blanche S, et al.: Efficacy and safety of darunavir/ritonavir at 48 weeks in treatment-naïve, HIV-1infected adolescents: Results from a phase 2 open-label trial (DIONE). Pediatr Infect Dis J 2014;33:940-945.

7. Tashima K, Crofoot G, Tomaka FL, et al:: Cobicistatboosted darunavir in HIV-1-infected adults: Week 48 results of a Phase IIIb, open-label single-arm trial. AIDS Res Ther 2014;11:39.

8. Mills A, Crofoot G Jr, McDonald C, et al:: Tenofovir alafenamide versus tenofovir disoproxil fumarate in the first protease inhibitor-based single-tablet regimen for initial HIV-1 therapy: A randomized phase 2 study. J Acquir Immune Defic Syndr 2015;69:439-445.

9. Lathouwers E, Wong EY, Luo D, Seyedkazemi S, De Meyer S, Brown K: HIV-1 resistance rarely observed in subjects using darunavir once-daily regimens across clinical studies. HIV Clin Trials 2017;18:196-204.

10. Eron J, Orkin C, Gallant J, et al.: A week 48 randomized phase 3 trial of darunavir/cobicistat/emtricitabine/tenofovir alafenamide in treatment-naïve HIV-1 patients. AIDS 2018;32:1431-1442.

11. Orkin C, Molina J-M, Negredo E, et al.: Efficacy and safety of switching from boosted protease inhibitors plus emtricitabine/tenofovir disoproxil fumarate regimens to the once-daily complete HIV-1 regimen of darunavir/cobicistat/ emtricitabine/tenofovir alafenamide $(\mathrm{D} / \mathrm{C} / \mathrm{F} / \mathrm{TAF})$ in viro- logically suppressed, HIV-1-infected adults through 48 weeks (EMERALD): A phase 3, randomized, non-inferiority trial. Lancet HIV 2018;5:e23-e34.

12. SYMTUZA ${ }^{\mathrm{TM}}$ (darunavir, cobicistat, emtricitabine, and tenofovir alafenamide) tablets Summary of Product Characteristics: Janssen-Cilag International NV, 2017. (Revised: July 2019). Available at www.medicines.org.uk/emc/product/ 8430, accessed October 7, 2019.

13. Prescribing information for SYMTUZA ${ }^{\mathrm{TM}}$ (darunavir, cobicistat, emtricitabine, and tenofovir alafenamide) tablets: Janssen Pharmaceutical Companies, 2018. (Revised: May 2019). Available at www.janssenlabels.com/package-insert/ product-monograph/prescribing-information/SYMTUZA-pi .pdf, accessed October 7, 2019.

14. Wensing AM, Calvez V, Gunthard HF, et al:: Update of the drug resistance mutations in HIV-1: June/July 2014. Top Antivir Med 2014;22:642-650.

15. De Meyer S, Vangeneugden T, van Baelen B, et al:: Resistance profile of darunavir: Combined 24-week results from the POWER trials. AIDS Res Hum Retroviruses 2008; 24:379-388.

16. Wensing AM, Calvez V, Günthard HF, et al.: 2015 Update of the drug resistance mutations in HIV-1. Top Antivir Med 2015;23:132-141.

17. Wensing AM, Calvez V, Günthard HF, et al.: 2017 Update of the drug resistance mutations in HIV-1. Top Antivir Med 2017;24:132-133.

18. Stanford University HIV drug resistance database. Available at https://hivdb.stanford.edu/dr-summary/resistancenotes/NRTI, accessed October 7, 2019.

19. DeJesus E, Ramgopal M, Crofoot G, et al.: Switching from efavirenz, emtricitabine, and tenofovir disoproxil fumarate to tenofovir alafenamide coformulated with rilpivirine and emtricitabine in virally suppressed adults with HIV-1 infection: A randomised, double-blind, multicentre, phase $3 \mathrm{~b}$, non-inferiority study. Lancet HIV 2017;4:e205-e213.

20. Orkin C, DeJesus E, Ramgopal M, et al: Switching from tenofovir disoproxil fumarate to tenofovir alafenamide coformulated with rilpivirine and emtricitabine in virally suppressed adults with HIV-1 infection: A randomised, double-blind, multicentre, phase $3 \mathrm{~b}$, non-inferiority study. Lancet HIV 2017;4:e195-e204.

21. Mills A, Arribas JR, Andrade-Villanueva J, et al.: Switching from tenofovir disoproxil fumarate to tenofovir alafenamide in antiretroviral regimens for virologically suppressed adults with HIV-1 infection: A randomised, active-controlled, multicentre, open-label, phase 3, noninferiority study. Lancet Infect Dis 2016;16:43-52.

22. Margot N, Cox S, Das M, McCallister S, Miller MD, Callebaut C: Rare emergence of drug resistance in HIV-1 treatment-naïve patients receiving elvitegravir/cobicistat/ emtricitabine/tenofovir alafenamide for 144 weeks. J Clin Virol 2018;103:37-42.

23. Sax PE, Wohl D, Yin MT, et al.: Tenofovir alafenamide versus tenofovir disoproxil fumarate, coformulated with elvitegravir, cobicistat, and emtricitabine, for initial treatment of HIV-1 infection: Two randomised, double-blind, phase 3, non-inferiority trials. Lancet 2015;385:2606-2615.

24. Sax PE, Pozniak A, Montes ML, et al.: Coformulated bictegravir, emtricitabine, and tenofovir alafenamide versus dolutegravir with emtricitabine and tenofovir alafenamide, for initial treatment of HIV-1 infection (GS-US-380-1490): A randomised, double-blind, multicentre, phase 3, noninferiority trial. Lancet 2017;390:2073-2082. 
25. Gallant J, Lazzarin A, Mills A, et al: : Bictegravir, emtricitabine, and tenofovir alafenamide versus dolutegravir, abacavir, and lamivudine for initial treatment of HIV-1 infection (GS-US-380-1489): A double-blind, multicentre, phase 3, randomised controlled noninferiority trial. Lancet 2017;390:2063-2072.

26. Daar E, DeJesus E, Ruane P, et al.: Efficacy and safety of switching to fixed-dose bictegravir, emtricitabine, and tenofovir alafenamide from boosted protease inhibitor-based regimens in virologically suppressed adults with HIV-1: 48 week results of a randomised, open-label, multicentre, phase 3, non-inferiority trial. Lancet HIV 2018;5:e347e356.

27. Molina JM, Ward D, Brar I, et al:: Switching to fixed-dose bictegravir, emtricitabine, and tenofovir alafenamide from dolutegravir plus abacavir and lamivudine in virologically suppressed adults with HIV-1: 48 week results of a randomised, double-blind, multicentre, active-controlled, phase 3, non-inferiority trial. Lancet HIV 2018;5:e357e365.

28. Llibre JM, Hung C-C, Brinson C, et al.: Efficacy, safety, and tolerability of dolutegravir-rilpivirine for the maintenance of virological suppression in adults with HIV-1: Phase 3, randomised, non-inferiority SWORD-1 and SWORD-2 studies. Lancet 2018;391:839-849.

29. Trottier B, Lake JE, Logue K, et al.: Dolutegravir/abacavir/lamivudine versus current ART in virally suppressed patients (STRIIVING): A 48-week, randomized, non-inferiority, open-label, phase IIIb study. Antivir Ther 2017;22:295-305.

30. Taiwo BO, Marconi V, Berzins B, et al.: Dolutegravir Plus Lamivudine Maintains Human Immunodeficiency Virus-1 Suppression Through Week 48 in a Pilot Randomized Trial. Clin Infect Dis 2018;66:1794-1797.

31. Joly V, Burdet C, Landman R, et al.: Dolutegravir and lamivudine maintenance therapy in HIV-1 virologically suppressed patients: results of the ANRS 167 trial (LAMIDOL). J Antimicrob Chemother 2019;74:739-745.

32. Perez-Molina JA, Rubio R, Rivero A, et al.: GESIDA 7011 study group. Dual treatment with atazanavir-ritonavir plus lamivudine versus triple treatment with atazanavir-ritonavir plus two nucleos(t)ides in virologically stable patients with HIV-1 (SALT): 48 week results from a randomised, openlabel, non-inferiority trial. Lancet Infect Dis 2015;15:775784.

33. Di Giambenedetto S, Fabbiani M, Quiros Roldan E, et al.: Treatment simplification to atazanavir/ritonavir + lamivudine versus maintenance of atazanavir/ritonavir + two NRTIs in virologically suppressed HIV-1-infected patients: 48 week results from a randomized trial (ATLAS-M). J Antimicrob Chemother 2017;72:1163-1171.

34. Huhn GD, Eron JJ, Girard PM, et al.: Darunavir/cobicistat/ emtricitabine/tenofovir alafenamide in treatment-experienced, virologically suppressed patients with HIV-1: subgroup analyses of the phase 3 EMERALD study. AIDS Res Ther 2019; $16: 23$.

35. Zaccarelli M, Santoro MM, Armenia D, et al.: Genotypic resistance test in proviral DNA can identify resistance mutations never detected in historical genotypic test in patients with low level or undetectable HIV-RNA. J Clin Virol 2016;82:94-100.

36. Gantner P, Morand-Joubert L, Sueur C, et al.: Drug resistance and tropism as markers of the dynamics of HIV-1 DNA quasispecies in blood cells of heavily pretreated patients who achieved sustained virological suppression. J Antimicrob Chemother 2016;71:751-761.

37. Huhn GS, Crofoot G, Ramgopal M, et al:: Darunavir/ cobicistat/emtricitabine/tenofovir alafenamide rapid initiation for HIV-1 infection. Primary analysis of the DIAMOND study. Presented at: American Conference for the Treatment of HIV; April 11-13, 2019. Miami

Address correspondence to: Erkki Lathouwers

Janssen Pharmaceutica NV

Turnhoutseweg 30 2340 Beerse Belgium

E-mail: elathouw@its.jnj.com 